\title{
Debate
}

\section{See and Treat: a management driven method of achieving targets or a tool for better patient care? One size does not fit all}

\author{
A M Leaman
}

We were very grateful to Leaman for his contribution that forms the first part of this debate. He is voicing concerns of many on the front line of A\&E feel and we acknowledge his forthright and courageous approach. Equally it can be easy to criticise but difficult to be constructive, especially in such an arduous task as improving the UK emergency care system. We therefore have invited responses to the issues raised in the hope of stimulating debate on these critical issues for emergency care in the UK. We thank all the authors in this section for their hard work and well argued contributions to this debate.

JW/PD

A $M$ Leaman Princess Royal Hospital NHS Trust, Telford TF1 6TF, UK

Correspondence to: Dr A M Leaman; shirley.redfern@prh.nhs.uk
- or anyone still uncertain about the concept, - See and Treat is a process whereby patients with minor conditions are seen soon after they arrive in A\&E by a senior clinician. Providing they have an appropriate problem such patients are given their definitive treatment straightaway and can then be discharged.

The Department of Health is very anxious that all A\&E departments should adopt See and Treat and has organised a road show that has toured the UK spreading the word. Circulars and emails, arriving on an almost daily basis, have encouraged senior A\&E staff to attend these meetings. One recent DoH letter even asked departments when, not whether, they would be introducing See and Treat.

The star turn at the See and Treat road show is the Kettering A\&E Department. Kettering had its Eureka moment when it found that it's throughput times were rising because all its cubicles (both major and minor) were blocked by patients waiting for admission. With the encouragement of its chief executive the A\&E departments response was to employ its most senior doctors and nurses in offices close to the waiting room where they found they could See and Treat quite a lot of patients with minor problems. This improved their department's deteriorating throughput times.

Many people might think that the proper response of the chief executive in this situation should have been to ask why his A\&E department was being blocked by patients waiting for a bed. Furthermore, how representative is the A\&E department in Kettering and are its solutions appropriate for more successful A\&E departments? Is it appropriate for the most experienced A\&E staff to see the patients with the least serious conditions?

The Department of Health's uncritical promotion of the See and Treat concept is wrong and should be questioned by $\mathrm{A} \& \mathrm{E}$ specialists

These considerations do not seem to have bothered the DoH, which, impressed by a few months improving figures, has decided that all departments should adopt the Kettering model. There has been no critical analysis of See and Treat. Nor have its principles been appraised in print by a major journal. Such disregard for scientific assessment is unfortunately all too prevalent in government circles. It should not however prevent
A\&E specialists from questioning the See and Treat concept.

Interestingly, much of the present waiting time problem is due to another concept that was introduced with little evaluation-triage. In particular the Manchester Triage Scale, with its notorious category 5 (or "wait until everyone else has been seen"), has encouraged excessive waiting times for those with less serious complaints.

In addition, $A \& E$ as a specialty has only itself to blame if it is now being told by others how to run its affairs. It was apparent at least 10 years ago that the chaos in most A\&E departments could not go on but senior A\&E figures failed to tackle this serious problem.

Before See and Treat is more widely introduced those organisations representing A\&E in the UK should demand more evidence. An interesting study at Kettering would be to give the A\&E department back its minor cubicles and to get the senior clinicians to use these spaces to see patients in the usual way. Would this produce a similar improvement in waiting times?

More information is needed about Kettering and the other exemplar A\&E departments. Were their waiting times originally worse than average? Do they have above average numbers of GP type attenders for whom See and Treat can be used? Can their improved times be sustained or do the senior clinicians "burn out"? What has been the impact on SHO training? Will See and Treat encourage patients with GP type problems to attend A\&E?

If the DoH really wants to help A\&E departments most would be greatly assisted by an end to trolleys being occupied by patients waiting for admission. Minimal delays for radiographs would also speed throughput. Queuing theory and other business concepts may have lessons for those running A\&E departments. However, patients, even those with minor complaints, are not "units" and should not be treated in this way. Such an approach is contrary to the holistic and personalised care to which most A\&E specialists aspire.

The Manchester triage category 5 should be abandoned. Such patients should be redirected to GP cooperatives, of which there are now many excellent examples. However, triage should not be completely abandoned. In addition to prioritisation, well trained triage nurses can start and complete many treatments, redirect GP type patients, and postpone minor A\&E cases if waiting times become excessive. 


\section{Don't throw triage out with the bathwater}

\section{J Windle, K Mackway-Jones}

$\mathrm{T}$ he concept of See and Treat has been heralded as something new and innovative that will ease the plight of emergency departments throughout England. However, anyone who has been in emergency care over the past 20 years will recognise this process as the norm during the early 1980s. Indeed triage was introduced to clinically risk manage this system of first come first serve and to re-direct the focus of scarce nursing and medical staff away from the most minor of presentations. For those who do remember these times there must have been a strong sense of déjà vu during their See and Treat workshop.

The Department of Health workshops focused on a series of exemplar hospitals who have introduced See and Treat. It is of note that they all appear to have a number of similarities, namely:

- streaming for major and minor patients did not exist before introducing See and Treat

- the waiting times did not appear excessive before See and Treat

- the case mix appears skewed to more minor cases

It would be appropriate to put this information clearly into the public domain so that others can see how close the situation in these hospitals is to their own. Once this is done it will be easier to judge how much of the apparent benefit can be attributed to streaming as compared with See and Treat.

We agree with Leaman who describes blocked beds and long trolley waits and questions the appropriateness of diverting senior clinical staff away from more complex cases in underresourced and overstretched departments. It would be a great mistake to confuse See and Treat with a quality initiative. Let's hold it up for what it is-a means to meet targets! To achieve the average wait of 75 minutes without solving the underlying problems of flow and resource the easiest group to "See and Treat" and get back in the street, has been singled out. A more sensible and quality driven initiative designed to improve the journey for patients requiring admission to hospital would be welcome. Rapid access to beds for those requiring admission would be a far better investment for patients, and coincidentally would free considerable resource in emergency departments. Unfortunately this is not an easy option or a quick fix; it does however have benefits across the whole of healthcare delivery.

The lack of formal evaluation of See and Treat is inevitable as departments are pushed into "piloting" the system. The superficial measurable "benefits" are plain to see and enticing. A virtually empty waiting room, reduced complaints about waiting times, and the presence of senior doctors in the department. The clinical costs will be much more difficult to measure-delays in the management of the smaller number of seriously ill and injured and less supervision of clinicians dealing with such cases. Furthermore, we should not delude ourselves about how long See and Treat will remain a continuous activity for senior doctors? Not long is the probable answer as the doctors become either 'burnt out' with the constant pressure to speed up the consultation or they simply lose interest in the limited challenge of managing minor cases.

Triage will remain an important risk management tool until the day resources always meet demand

No discussion around See and Treat could be complete without rubbishing triage and Leaman has not let us down! Triage has received a great deal of bad press recently and been blamed for the long waiting times experienced by patients who have been assessed and deemed able to wait for treatment due to the minor nature of their presenting complaint. I have bad news, triage and indeed The Manchester Triage System (MTS) does not, has never, and will not reduce waits. This is not the purpose of triage. Triage was the first formalised system of clinical risk management, and it is surprising that in an era of Clinical Governance and CHI reviews this simple fact has not been recognised. Triage was designed to ensure that whenever demand exceeded resources (all the time in British emergency departments at present) then the limited resource was directed to the case with the greatest clinical need. It is inevitable that this will direct resources away from less urgent cases, however if we subscribe to the idea the most seriously ill and injured should receive the greatest resources, both human and environmental, then MTS priority reveals those patients safely and appropriately. The problem is not the triage priority or the system used to reach that decision, but the basic under-resourcing. In such a situation triage is just a messenger not a problem in itself.

The Manchester Triage Group would strongly reject the comment that MTS was introduced with little evaluation. While we acknowledge consensus view is the weakest form of evidence the process involved in developing the system took in excess of a 1000 person hours and that figure should be multiplied by the 20 strong group of contributors. The system was piloted and subjected to audit in the nine Manchester hospitals the system was originally developed for. This was before the group were asked to teach the system across the UK. Additionally the words "not evidence based" are often used here without an understanding that until we agree a "gold standard" method of determining how urgent conditions are, it is not possible to prove that any system works. The MTS is an attempt to break this chicken and egg conundrum, and should provide a starting point for future changes.

The final paragraph of the paper throws up the issue of "the notorious category 5 patients". If the author were well versed with MTS he might realise just how few patients could actually meet the

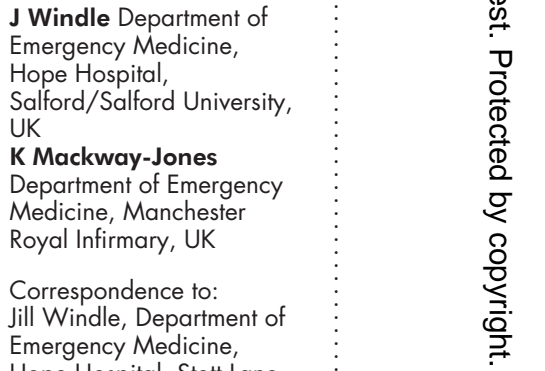
Hope Hospital, Stott Lane, Salford M6 8HN, UK; jill.windle@srht.nhs.uk 


\section{Debate}

criteria for this non-urgent category. MTS prioritises all patients with a recent complaint (defined as within seven days) in category four, along with any with mild pain. As these discriminators describe two of the most likely reasons to seek medical attention there are few patients left for category 5 . Instead of using the blue category as it was developed (to define patients who have conditions that are stable enough to wait for assessment) it has been abused to try and restrict access for patients deemed "inappropriate" in the emergency department, and surprisingly to define those who are "primary care".

The plea for triage to remain as a means of assessing and prioritising patients is heartening but the idea of increasing the amount of activity at triage may contribute to the long waits in the first place. Many units report waits of over 40 minutes to be triaged. This seems incomprehensible, and dangerous, as the queue has merely shifted from the waiting room to the triage room. The difference in this situation is of course the waiting room queue has been assessed and prioritised, the triage queue is an unknown entity.

Few would disagree that the reconfiguration of emergency services requires a whole systems approach, of which See and Treat is just one element. If there are important benefits to See and Treat then let us evaluate them but also examine the effect on the rest of the department. Armed with this information, departments will be in a much better position to consider the options and make the system "best fit". It would hopefully also put a stop to the currently adopted herding instinct, where we run from one new idea to another as changing is always better than staying the same. In the mean time triage will remain an important clinical risk management tool, and will continue in this role until the utopian day when resources finally match demand.

\section{One size does not fit all. View 2}

\section{K Castille, M Cooke}

$\overline{\mathrm{i}}$ he advantage of having senior clinicians seeing and dealing with patients at the earliest possible opportunity is virtually uncontested across a wide spectrum of healthcare services. The concept of See and Treat is entirely based on this premise, and it is therefore not surprising that it works. The benefits of See and Treat, as part of an emergency department system are clear:

- Significant reductions in both the time that each patient has to wait and the total number of patients waiting at any one time

- Improvement in both patient experience and staff job satisfaction (concerns about staff job satisfaction emanate from those who have not tried it, rather than those who have)

Leaman offers a good description of See and Treat. His criticism seems to be mostly confined to the reasons for its introduction. We have therefore focused our response on his five main points which, in summary, appear to be that See and Treat:

l was instigated by a Trust chief executive in response to deteriorating throughput times in A\&E

2 was initiated as the incorrect response to bed blocking

K Castille Director of Emergency Sevices, NHS Modernisation Agency, UK M Cooke A\&E advisor, Department of Health, UK

Correspondence to: Karen Castille, NHS Modernisation Agency, Richmond House, 79 Whitehall, London SWIA 2NS, UK; karen.castille@tesco.net
The author concludes that See and Treat should not be introduced and that, instead, the solutions lie in abandoning Manchester triage category 5; using triage nurses to carry out treatments; and postponing minor A\&E "cases" when waiting times become excessive.

We will briefly consider each of the criticisms and evaluate the conclusion drawn.

The first of the five points is factually incorrect. The chief executive, while supportive, had no direct involvement in the introduction of the See and Treat system. It was devised and implemented by the A\&E consultant and her clinical team, resulting in a demonstrable improvement in the patients' waiting times. ${ }^{1}$ A key feature of See and Treat, as both a concept and a working system, is that it originated, and continues to be developed and tested by clinicians. It is insulting to clinicians to suggest that they might have responded to "being told by others what to do".

Other emergency departments have introduced systems with senior clinicians assessing and treating patients. There are also examples of clinicians implementing similar principles in America $^{2}$ and New Zealand. ${ }^{3}$

Interestingly, about $20 \%$ of A\&E departments are known to have introduced some form of See and Treat before the Modernisation Agency's workshops were held. Hence the clinician to clinician networks were already in operation. The role of the NHS Modernisation Agency is to facilitate and support such ventures by enabling clinicians and managers to work together and find solutions that are workable, and beneficial for patients.

The second criticism is that the issues that need to be tackled lie outside of A\&E, so why are we concentrating on patients with minor problems? 
We agree that there are a number of issues that need to be tackled outside of A\&E and to this end the Emergency Services Collaborative (ESC) has been established to tackle waits and delays across the whole system of emergency care. See and Treat constitutes only a small part of this work. The organisations involved in the first wave of the ESC are already helping to develop both local and national plans to tackle the complex problems related to patient flows, such as improved bed management. ${ }^{4}$ This is being done with the whole system in mind. Furthermore, one of the principles behind See and Treat-getting senior expertise at the front end of the patient episode-can be applied to patient flows where the patient has more serious and complex problems. This has been shown to benefit patients. ${ }^{5}$ Specifically, the impact of the use of a clinical decision unit (Has$\operatorname{san}^{6}$ ) and the review of the role of an assessment ward $^{7}$ are highlighted elsewhere in this edition of EMJ.

See and Treat is therefore only one aspect of the work of the ESC. It can be introduced comparatively quickly and effectively compared with some of the more complex issues that surround bed management. See and Treat is not the solution to all emergency care problems. However, it does provide the opportunity for A\&E clinicians to exercise control over the issues that are within their direct domain.

With regard to the third point, guiding principles for the introduction of See and Treat were published in October 2002. ${ }^{8}$ These principles were collated from all the known existing models of See and Treat. A consensus group, of experienced A\&E clinicians, was used to draft the principles, which were further developed and endorsed by the RCN and BAEM. There are several published studies that have described the impact of the application of similar principles in improving patient waiting times without detriment to major injury patients. ${ }^{3910}$ There are also examples from experiences in UK emergency patients. ${ }^{11}{ }^{12}$

The NHS Modernisation Agency has recently completed a series of national workshops in which a variety of different models of See and Treat were presented by clinicians from across the country. The Kettering model was one of a variety of examples presented to show how clinicians had tackled the issues in different ways, and in response to local needs. Hence, we promote the importance of designing a local model to meet local needs.

It is important to emphasise that See and Treat is not regarded to be the domain of A\&E consultants alone. The author endorses and speaks positively about "well trained triage nurses who can initiate and complete many treatments" - this is, in fact, a model of See and Treat. Most of the known models use a combination of team members to deliver the most appropriate service to meet patients' needs. The focus should be on competence and skills and not professional backgrounds or job titles.

The issue of evidence based practice is raised and the author emphasises that there should be published evidence for all of the ways in which we work. However, it is interesting that he uses no references to support his concluding suggestion to "abandon Manchester Triage Scale category 5". There is an increasing amount of evidence to sup- port the principles of See and Treat. This evidence is derived from empirically tested, clinician led local change. It also draws on historical evidence and data from service provision. Professional consensus has also played a large part in its development. Hence, this triangulated approach is based on pragmatic science. Although not level one evidence, it is a stronger evidence base than can be applied to the current systems. The unanimous experience of those who have tried a model of See and Treat is that it reduces waiting times and improves both patient and staff experience, in ways that the other options do not. Therefore See and Treat has a stronger evidence base than maintaining the status quo, or introducing an untested hybrid model. The lack of high level evidence for See and Treat is not an adequate reason to maintain existing systems that have developed through tradition, with even less evidence base.

The last concern is that applying modern operations management theory to health care is problematic because it depersonalises care. The advantages of this approach are detailed in this edition of EMJ by Walley. ${ }^{13}$ The question is, whether developing an effective system for all patients makes it worse for individual patients? In reality there is no trade off, because the function of the system is to provide the best care for each and every individual. See and Treat allows personalised, timely and effective care to be delivered for large numbers of patients, who previously were enduring a very poor experience of care. It is a strange and inaccurate observation to imply that this makes care less, rather than more, personal. This is reinforced by a recent poll on emergency departments (MORI 2002, unpublished), which showed that the main concern of patients was waiting time and, in particular, waits for treatment of minor injuries.

We do not believe that triage should be abandoned in itself. However, when See and Treat is functioning optimally, there is no need to prioritise care (triage).

In conclusion, See and Treat is a system of care delivery that has been developed by clinicians and is based on sound operational principles. It is not based on a single prescriptive model. On the contrary, clinicians across the country have adapted it to suit their local services and the needs of their patients. It is delivered through a set of principles that were first developed by clinicians, and then further adapted by BAEM and the RCN. Moreover, John Heyworth president of BAEM, and Lynda Holt Chair of the RCN A\&E Nursing Association, wrote the endorsing foreword in the guiding principles.

See and Treat enables us to care effectively for a large group of A\&E patients, and is-so far-the best alternative we have. We could however decide to retain the current system, which manifests as full waiting rooms and the ensuing oppression that this induces in our A\&E departments. Alternatively we can choose to actively take steps to improve the current system. See and Treat provides us with such an opportunity.

\section{REFERENCES}

1 Shrimpling $\mathbf{M}$. Redesigning triage to reduce waiting times. Emergency Nurse 2002;10:34-7.

2 Partovi SN, Melson BK, Bryan ED, et al. Faculty triage shortens emergency department length of stay. Acad

Emerg Med 2001;10:990-5.
Debate 
3 Ardagh MW Wells JE, Cooper K, et al. Effect of a rapid assessment clinic on waiting time to be seen by a doctor and time spent in the department, for patients presenting to an urban emergency department: a controlled prospective trial. N Z Med J 2002;115:U28.

4 Department of Health. A good practice guide on anticipatory management in healthcare. London: Department of Health, 2002. (nww.doh.nhsweb.uk/nhs/ hoip/index.htm)

5 Wanklyn P, Hosker $\mathrm{H}$, Pearson $\mathrm{S}$, et al. Slowing the rate of acute medical admissions. J $R$ Coll Physicians 1997; $31: 173-6$

6 Hassan TB. Clinical decision units in the emergency department: old concepts, new paradigms, and refined gate keeping. Emerg Med J 2003;20: 123-5.

7 MW Cooke, Higgins J, Kidd P. Use of emergency

observation and assessment wards: a systematic literature review. Emerg Med J 2003;20:138-42.
8 Anon. See and Treat. London: NHS Modernisation Agency, 2002. (www.modern.nhs.uk/emergency) 9 Redmond AD, Buxton N. Consultant triage of minor cases in an accident and emergency department. Arch Emerg Med 1993;10:328-30.

10 Partovi SN, Melson BK, Bryan ED, et al. Faculty triage shortens emergency department length of stay. Acad Emerg Med 2001;10:990-5.

11 Cooke MW, Wilson S, Pearson S. The effect of a separate stream for minor injuries on accident and emergency department waiting times. Emerg Med J 2002;19:28-30

12 Anon. Emergency Services Collaborative-case studies. London: Modernisation Agency, 2002

(www.modern.nhs.uk/emergency)

13 Walley P. Designing the A\&E system: lessons from manufacturing. Emerg Med J 2003;20:126-30 